\title{
Role of NO pathway, calcium and potassium channels in the peripheral pulmonary vascular tone in dogs
}

\author{
F. Chabot, F. Schrijen, C. Saunier
}

\begin{abstract}
Role of NO pathway, calcium and potassium channels in the peripheral pulmonary vascular tone in dogs. F. Chabot, F. Schrijen, C. Saunier. (C) ERS Journals Ltd 2001. ABSTRACT: Because hypoxic pulmonary vasoconstriction occurs mainly in the small pulmonary arteries, the authors investigated the effects of drugs acting on the nitric oxide (NO) pathway and the calcium and potassium channels in the peripheral pulmonary circulation, without interference with the overall pulmonary or systemic circulation.

Mixed venous blood was infused in wedged areas to study the pressure/flow relationship and to compute peripheral pulmonary vascular resistance (PPVR). The authors studied the effects of $\boldsymbol{N}^{\omega}$-nitro-L-arginine methyl ester (L-NAME), an NO synthase inhibitor, sodium nitroprusside (SNP, an NO donor), the calcium channel blockers verapamil, nifedipine and nicardipine, and the potassium channel opener levcromakalim, during normoxia and acute mild normocapnic hypoxia.

In the peripheral pulmonary circulation, L-NAME caused an increase in PPVR during normoxia $(+95 \% ; p<0.001)$ and hypoxia $(+60 \% ; p<0.01)$. Following the increase by L-NAME, SNP decreased PPVR during normoxia $(-24 \% ; p<0.05)$ and hypoxia $(-23 \% ; \mathbf{p}<0.05)$. Verapamil, nifedipine and nicardipine did not modify PPVR during normoxia but during hypoxia they decreased PPVR $(-28 \%$, nonsignificant; $-27 \%$, $\mathbf{p}<0.01$ and $-33 \%, p<0.05$, respectively). Levcromakalim did not modify PPVR during normoxia or hypoxia.

In conclusion, the nitric oxide pathway and voltage-dependent calcium channels, and not adenosine triphosphate sensitive potassium channels, play an important role in the control of peripheral pulmonary circulation in dogs.
\end{abstract}

Eur Respir J 2001; 17: 20-26.

INSERM U14, Plateau de Brabois, C.O. 10, Vandœuvre-les-Nancy and Service des Maladies Respiratoires et Réanimation Respiratoire, $\mathrm{CHU}$ Nancy-Brabois, Vandœuvre-les-Nancy, France.

Correspondence: F. Chabot, Service des Maladies Respiratoires et Réanimation Respiratoire, CHU Nancy-Brabois, rue du Morvan, 54500, Vandœuvre-les-Nancy, France. Fax: 330383154023

Keywords: Anaesthetized dogs ATP-sensitive potassium channel opener calcium channel inhibitors

hypoxia

peripheral pulmonary circulation pulmonary vasoreactivity

Received: May 192000

Accepted after revision September 15 2000
Segmental differences in vasomotor reactivity are well documented in the pulmonary vasculature. Hypoxia causes sustained constriction in resistance pulmonary arteries [1] while causing a biphasic response in conduit pulmonary arteries [2]. Investigations on resistance vessels have been performed mainly in vitro on small isolated pulmonary artery rings. Some authors investigated the relationship between pressure and flow in the pulmonary circulation in vivo for a lobe in situ [3], but the preparation included predominantly conduit vessels and the flow changes induced modifications of other variables. Because alveolar hypoxia is an important regulator of pulmonary vascular tone and causes vasoconstriction mainly in small pulmonary arteries, the authors studied, in vivo, the pharmacology of the vasomotor response of the peripheral pulmonary circulation, during normoxia and mild acute hypoxia.

Under physiological conditions, the vasodilator nitric oxide (NO) is continually released by endothelial cells and regulates organ and perfusion pressure and flow [4]. Endogenous NO may contribute to the maintenance of normal pulmonary vasomotor tone. Pulmonary vascular tone is also regulated by the activity of calcium and potassium channels, and the links between activation of both calcium and potassium channels and
NO-induced relaxation in the proximal part of the pulmonary artery have been emphasized, in vitro [5]. Various inhibitors of NO synthase (NOS) have been shown to increase the normoxic pulmonary vascular tone in some [6, 7] but not in all [8,9] experiments. The inhibition of NO synthesis increases hypoxic pulmonary vasoconstriction in intact lungs, suggesting an increased NO synthesis in response to hypoxia and/ or vasoconstriction [6].

The calcium and potassium channels seem also to play a role in the mechanism of hypoxic pulmonary vasoconstriction (HPV). Potassium channel blockers cause pulmonary vasoconstriction [10] largely through their effects on membrane potential and calcium channels. It has been proposed that hypoxia might inhibit outward potassium current in pulmonary smooth muscle cells, causing membrane depolarization and thus permitting calcium entry through the voltagedependent calcium channels sensitive to dihydropyridine [11]. Calcium channel blockers like verapamil, a phenylalkylamine, have been shown to inhibit HPV [12], but Young et al. [13] did not observe this inhibition with verapamil whereas nifedipine, a dihydropiridine, appeared to be a more effective pulmonary vasodilator. 
Studies of the peripheral pulmonary circulation are difficult to perform in vivo. The present authors studied the pressure/flow relationship in a small peripheral portion of the pulmonary vascular bed, excluding most of the large conduit arteries, where pressure could be increased by hypoxia or pharmacologically modified without influencing the rest of the circulation [14]. This technique was used to study, during normoxia or acute normocapnic hypoxia, the action of the NO pathway by the administration of sodium nitroprusside (SNP), an NO donor, or by inhibition of NOS by $N^{\omega}$-nitro-Larginine methyl ester (L-NAME). The role of calcium channels was studied by a blockade with verapamil, nifedipine and nicardipine, and the role of potassium channels by the administration of a potassium channel opener, levcromakalim, in the peripheral pulmonary vasculature.

\section{Material and methods}

All experiments were conducted according to the Helsinki convention for the care and use of animals.

\section{Study design}

In anaesthetized dogs breathing spontaneously, vasoactive drugs were infused in a distal portion of the lung vascular bed, and the pressure/flow relationship was determined to compute the peripheral pulmonary vascular resistance (PPRV). Isotonic glucose was used as control. The drugs were given while the dogs breathed either room air, or a hypoxic mixture $\left(\mathrm{O}_{2} 10 \%\right.$, $\mathrm{CO}_{2} 3 \%$, balance $\mathrm{N}_{2}$ ).

\section{Peripheral pulmonary vascular resistance model}

The system used has been described previously in detail [14]. Anaesthesia was induced with an initial dose of $20 \mathrm{mg} \cdot \mathrm{kg}^{-1}$ thiopental $i . v$., and maintained by a slowrate infusion $\left(14 \mathrm{mg} \cdot \mathrm{kg}^{-1} \cdot \mathrm{h}^{-1}\right)$, with an electrical syringe (Vial SE 400, Grenoble, France). The dogs were intubated with a cuffed tracheal cannula whilst breathing spontaneously. Under sterile conditions, a femoral artery was cannulated with a catheter, and an external jugular vein with two catheters: a conventional SwanGanz thermodilution catheter, with the proximal lumen opening at $20 \mathrm{~cm}$ from the tip, and a 7F custom-made balloon catheter with both lumens extending to the tip (part No 600518 model, American Edwards Laboratories, Santa Ana, CA, USA). Both catheters were advanced, under radiograph, pressure and electrocardiograph (ECG) monitoring, into the pulmonary artery. The thermodilution catheter tip was advanced until a wedge pressure could be obtained by balloon inflation (the proximal lumen was then in the right atrium). The double lumen catheter was advanced, in another pulmonary artery, until it wedged itself i.e. when the internal diameter of the vessel was equal to the external diameter of the catheter $(2.3 \mathrm{~mm})$.

Femoral arterial pressure was continuously monitored. Pulmonary arterial (PAP), pulmonary wedge (PWP), and right atrial (RAP) pressures, as well as cardiac output (CO), were measured with the Swan-Ganz catheter. Blood samples were withdrawn simultaneously from both femoral and pulmonary arterial catheters to determine arterial and mixed venous blood gases. To determine the pressure/flow curve, one lumen of the double lumen catheter was connected to a pressure transducer, the other one to a peristaltic pump (Ismatec, Zurich, Switzerland). Blood was withdrawn through the pump from the distal lumen of the thermodilution catheter, which was lying free in the pulmonary artery. Blood flow was monitored with a Transonic Systems (Ithaca, NY, USA) flowmeter. The catheters were primed with blood. Drugs or control solution (isotonic glucose $50 \mathrm{~g} \cdot \mathrm{L}^{-1}$ ), were administered with an electrical syringe (Vial SE 400, Grenoble, France) and added to the blood infusion, with a rate of infusion equal to $15 \%$ of that of the blood infusion.

To study the pressure/flow curves, blood flow was increased by steps from 0 to $\sim 5,8$ and $10 \mathrm{~mL} \cdot \mathrm{min}^{-1}$; each flow was maintained for $\geqslant 1 \mathrm{~min}$, and the pressure was measured after equilibration at the end of each period. The drugs were used at increasing concentrations.

After the pressure/flow runs had been completed, contrast medium was slowly infused into the wedged catheter, until the draining vein was visualized. The volume infused was taken as a measurement of the volume of the wedged area. The angiogram was always performed after the end of the pharmacological study since a previous study showed that contrast medium increased the PPVR [15].

At the end of the experiment, the special catheter was withdrawn, and blood was sampled from the pulmonary artery via the thermodilution catheter, in order to calibrate the flowmeter with a stop-watch and a graduated tube.

\section{Hypoxia}

The dogs breathed alternately, at random, room air or a hypoxic mixture $\left(\mathrm{O}_{2} 10 \%, \mathrm{CO}_{2} 3 \%, \mathrm{~N}_{2} 87 \%\right)$ administered from a bag through a nonrebreathing valve. Haemodynamic data were measured after $10 \mathrm{~min}$ hypoxic ventilation. During this time, blood flow was maintained in the wedged area, after which, the pressure/flow curve was determined. Before switching between normoxia and hypoxia, the double lumen catheter was wedged in another site. Different drugs were tested in different areas, except for sodium nitroprusside after L-NAME.

\section{Pharmacological studies}

The results were obtained from 96 experiments in $24 \mathrm{dogs}$, weighing $24.9 \pm 0.8 \mathrm{~kg}$ (mean \pm SEM). Seven protocols were performed, during normoxia and hypoxia. Control experiments were performed with isotonic glucose $\left(50 \mathrm{~g} \cdot \mathrm{L}^{-1}\right)$ repeated four times consecutively in the same area (protocol 1). In separate experiments, the effects of L-NAME $\left(10^{-7}, 10^{-5}\right.$ and $\left.10^{-3} \mathrm{M}\right)$ were studied (protocol 2). The effects of SNP $\left(10^{-7}, 10^{-5}\right.$ and $10^{-3} \mathrm{M}$ ) were investigated following L-NAME $\left(10^{-3} \mathrm{M}\right.$; protocol 
3). To assess the effects of calcium channel inhibitors, the effects of verapamil $\left(10^{-10}, 10^{-8}, 10^{-6} \mathrm{M}\right)$, nifedipine $\left(10^{-11}, 10^{-9}, 10^{-7} \mathrm{M}\right)$ and nicardipine $\left(10^{-10}, 10^{-8}, 10^{-6} \mathrm{M}\right)$ were studied (protocols 4,5 and 6 , respectively). In experiments designed to assess the role of potassium channel opening, the effects of levcromakalim $\left(10^{-8}\right.$, $10^{-6}$ and $10^{-4} \mathrm{M}$ ) were investigated (protocol 7).

\section{Drugs}

L-NAME and SNP were purchased from Sigma (Saint Quentin-Fallavier, France). Verapamil was obtained from Knoll (Levallois-Perret, France), nifedipine from Bayer (Leverkusen, Germany), and nicardipine from Sandoz (Basel, Switzerland). Levcromakalim was kindly supplied by Smith Klein Beecham (Worthing, UK). Drugs were solubilized in glucose $50 \mathrm{~g} \cdot \mathrm{L}^{-1}(\mathrm{~L}-$ NAME and SNP), in distilled water (levcromakalim), in alcohol and polyethylene glycol (nifedipine) and in hydrochloric acid (nicardipine). Then an aliquot of these solutions was dissolved in isotonic glucose (50 $\left.\mathrm{g} \cdot \mathrm{L}^{-1}\right)$ to obtain the various concentrations used. Control solutions were prepared from the solvents in the absence of active principle. The $\mathrm{pH}(\sim 6)$ and the viscosity of the control and drug solution were the same. All solutions were prepared just prior to use.

\section{Derived variables}

The perfusion pressure during the pressure/flow runs was taken as the difference between the actual wedge pressure and the wedge pressure measured at zero flow. Overall pulmonary vascular resistance $\left(\mathrm{PVR}, \mathrm{dyn} \cdot \mathrm{s} \cdot \mathrm{cm}^{-5}\right.$ ) was computed as $80 \times$ PAP-PWP; $\mathrm{mmHg}) / \mathrm{CO}\left(\mathrm{L} \cdot \mathrm{min}^{-1}\right)$. PPVR $\left(10^{3} \cdot \mathrm{dyn} \cdot \mathrm{s} \cdot \mathrm{cm}^{-5}\right)$ was determined applying the same formula to the local pressure/flow curve, with the perfusion pressure taken at $5 \mathrm{~mL} \cdot \mathrm{min}^{-1}$ flow [14].

\section{Statistical analysis}

In each protocol, differences between groups were tested by analysis of variance (ANOVA), differences between periods by paired t-tests, and relations between variables by least squares regression analysis [16]. All values are expressed as mean \pm SEM.

\section{Results}

The pressure/flow relationship was determined in 49 sites during normoxia and 47 other areas during hypoxia. The volume of the wedged area was $0.65 \pm 0.03 \mathrm{~mL}$ in air, and $0.61 \pm 0.04 \mathrm{~mL}$ during hypoxia. Blood gases during normoxia were within normal limits (table 1). During hypoxia, due to the presence of $\mathrm{CO}_{2}$ in the inspired mixture, $\mathrm{Pa}, \mathrm{CO}_{2}$ remained unchanged, $\mathrm{Pa}, \mathrm{O}_{2}$ fell, and ventilation volume doubled with increased breathing frequency. Haemodynamic data were within normal limits during normoxia. During hypoxia, pulmonary artery pressure and pulmonary vascular resistance increased significantly $(+20 \%$ and $+49 \%$, respectively). During the pressure/flow runs, heart rate, systemic arterial pressure, pulmonary arterial pressure
Table 1. - Blood gases, ventilation and haemodynamic data during normoxia and hypoxia

\begin{tabular}{lccc}
\hline & Normoxia & Hypoxia & $\mathrm{p}$ \\
\hline Subjects n & 49 & 47 & \\
$\mathrm{pH}$ & $7.34 \pm 0.01$ & $7.35 \pm 0.01$ & $\mathrm{NS}$ \\
$\mathrm{Pa}, \mathrm{CO}_{2} \mathrm{mmHg}$ & $37.3 \pm 0.5$ & $35.6 \pm 0.6$ & $\mathrm{NS}$ \\
$\mathrm{Pa}, \mathrm{O}_{2} \mathrm{mmHg}^{-1}$ & $84 \pm 1$ & $45 \pm 1$ & $\mathrm{NA}$ \\
$V^{\prime} \mathrm{L} \cdot \mathrm{min}^{-1}$ & $6.3 \pm 0.3$ & $14.0 \pm 0.6$ & $<0.001$ \\
$\mathrm{fR} \mathrm{c} \cdot \mathrm{min}^{-1}$ & $21 \pm 1$ & $28 \pm 2$ & $<0.01$ \\
$\mathrm{mSAP} \mathrm{mmHg}$ & $159 \pm 2$ & $165 \pm 3$ & $\mathrm{NS}$ \\
$\mathrm{HR} \mathrm{min}$ & $202 \pm 4$ & $200 \pm 4$ & $\mathrm{NS}$ \\
$\mathrm{CO} \mathrm{L} \cdot \mathrm{min}^{-1}$ & $4.9 \pm 0.2$ & $4.7 \pm 0.2$ & $\mathrm{NS}$ \\
$\mathrm{mPAP} \mathrm{mmHg}$ & $20 \pm 1$ & $24 \pm 1$ & $<0.001$ \\
$\mathrm{mPWP} \mathrm{mmHg}$ & $9 \pm 0.3$ & $9 \pm 0.3$ & $\mathrm{NS}$ \\
$\mathrm{PVR} \mathrm{dyn} \cdot \mathrm{s} \cdot \mathrm{cm}^{-5}$ & $181 \pm 10$ & $269 \pm 14$ & $<0.001$ \\
PPVR $10^{3} \cdot \mathrm{dyn} \cdot \mathrm{s} \cdot \mathrm{cm}^{-5}$ & $101 \pm 9$ & $126 \pm 10$ & $<0.05$ \\
\hline
\end{tabular}

Data are presented as mean \pm SEM. n: number of sites; $V^{\prime}$ : minute ventilation; $f \mathrm{R}$ : breathing frequency; mSAP: mean systemic arterial pressure; HR: heart rate; CO: cardiac output; mPAP: mean pulmonary artery pressure; mPWP: mean wedge pressure; PVR: pulmonary vascular resistance; PPVR: peripheral pulmonary vascular resistance; NS: nonsignificant; NA: not applicable.

and right atrial pressure did not change. No significant change in haemodynamic variables, including $\mathrm{CO}$ and cardiac filling pressures was induced by the pharmacological studies.

\section{Protocol 1: effects of isotonic glucose}

PPVR, computed from four consecutive curves with isotonic glucose, showed no difference with time during normoxia $(n=8)$ or hypoxia $(n=4)$.

\section{Protocols 2-3: effects of NOS inhibition and SNP}

L-NAME increased PPVR under normoxia $(n=5)$ and hypoxia $(\mathrm{n}=10)$, and with the highest concentration, PPVR reached the same value in the two conditions (fig. 1). The dose-response correlation was

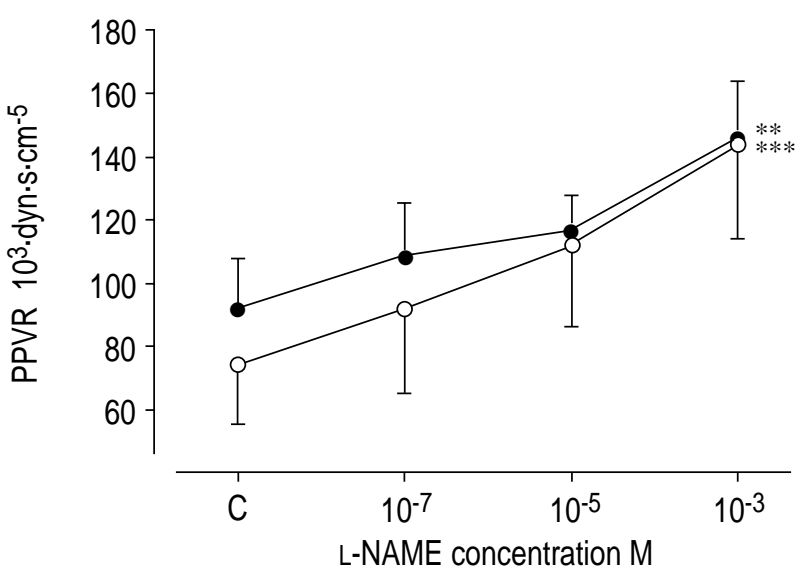

Fig. 1. - Effect of L-NAME on peripheral pulmonary vascular resistance (PPVR) during normoxia $(O)$ and during hypoxia (O). Results are expressed as mean \pm SEM. C: control. A statistically significant difference was found by two-way analysis of variance for the responses of PPVR to L-NAME during normoxia $(* * *: p<0.001)$ and during hypoxia $(* *: p<0.01)$ 
a)

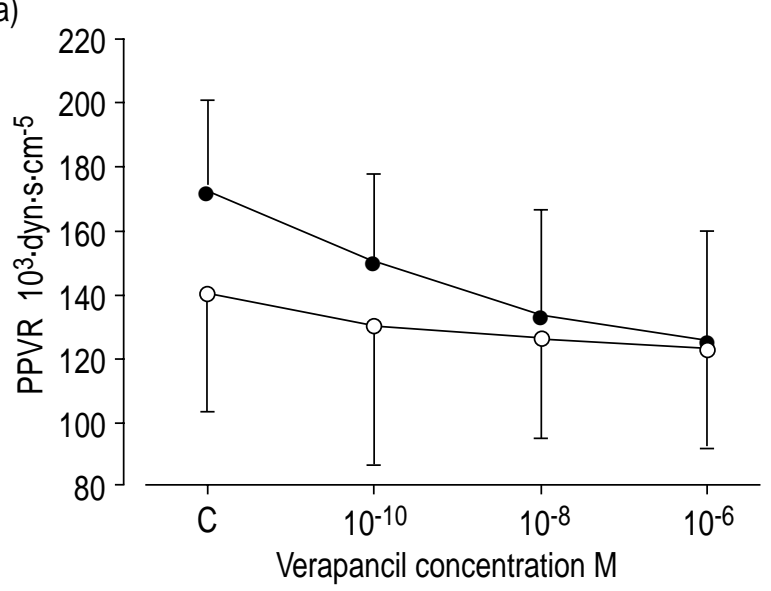

c)

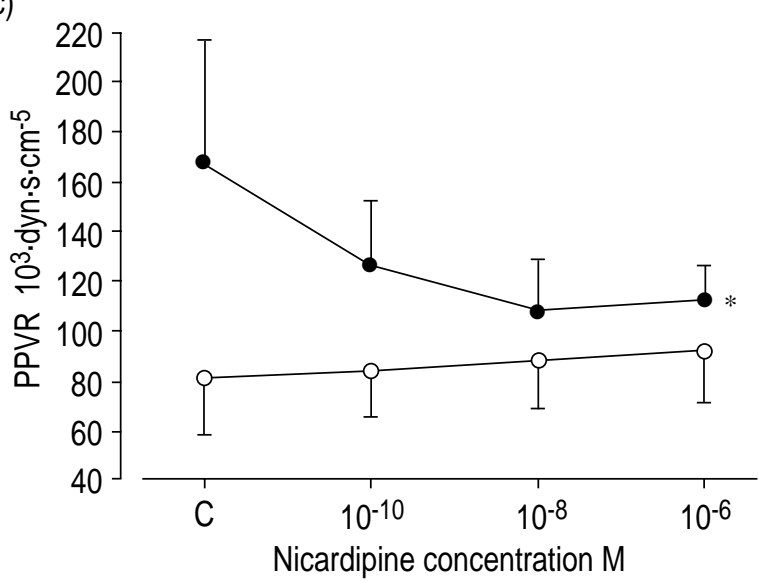

b)

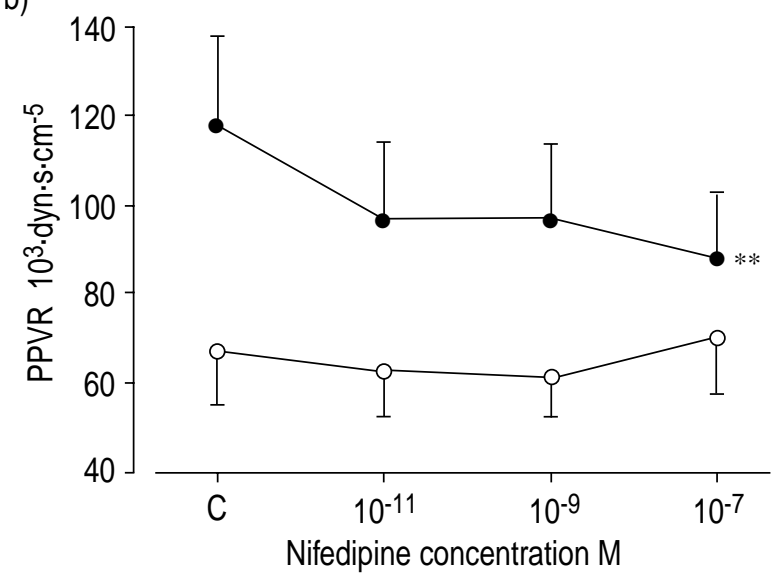

d)

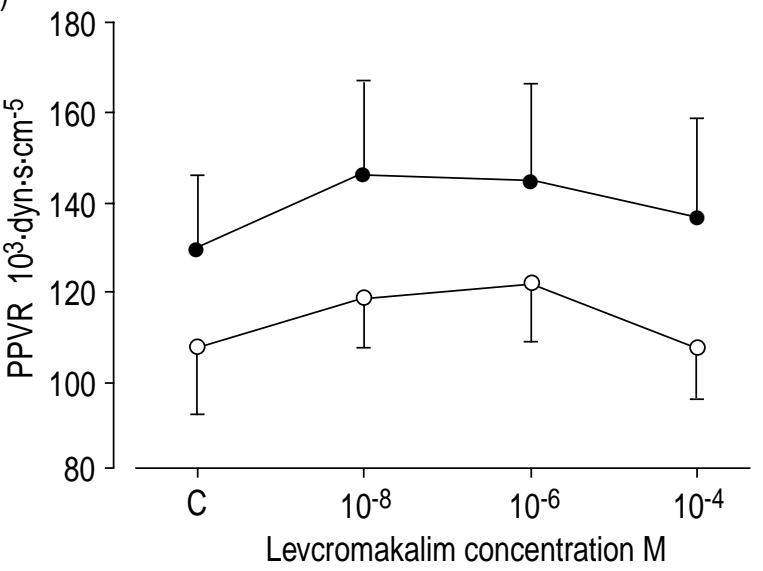

Fig. 2. - Effects of calcium channel blockers and levcromakalim on peripheral pulmonary vascular resistance (PPVR) in air ( $\bigcirc)$ and during acute hypoxia $(\mathbf{O})$. a) verapamil; b) nifedipine; c) nicardipine; d) levcromakalim. Data expressed as mean \pm SEM. C: control; **: $\mathrm{p}<0.01$ found by two-way analysis of variance (ANOVA) between responses of PPVR from control to nifedipine at $10^{-7} \mathrm{M}$ during hypoxia; *: $\mathrm{p}<0.05$ found by two-way ANOVA between responses from control to nicardipine at $10^{-6} \mathrm{M}$ during hypoxia.

significant in normoxia and in hypoxia $(\mathrm{p}<0.001$ and $\mathrm{p}<0.01$, respectively). After an increase in PPVR with L-NAME $\left(10^{-3} \mathrm{M}\right)$, perfusion of SNP at $10^{-7}, 10^{-5}$ and $10^{-3} \mathrm{M}$, induced a significant decrease in PPVR, during normoxia $(n=5 ; p<0.05)$ and hypoxia $(n=4 ; p<0.05)$.

\section{Protocols 4-6: effects of calcium channel blockers}

During normoxia, verapamil $(n=5)$, nifedipine $(n=9)$, or nicardipine ( $n=7$; fig. 2 ) did not change PPVR. During normocapnic hypoxia, verapamil tended to cause a decrease in PPVR $(n=5, p=0.07)$ and nifedipine caused a significant decrease in PPVR $(n=5, p<0.01)$. The changes with nicardipine were of the same kind as with nifedipine: during hypoxia, PPVR decreased by $33 \%(n=9, p=0.04)$.

Protocol 7: effects of potassium channel opener levcromakalim

As shown in figure 2, levcromakalim, from $10^{-8}$ to $10^{-4} \mathrm{M}$, did not induce a significant change in PPVR, during normoxia $(n=10)$ or hypoxia $(n=9)$. PPVR baseline values, obtained with control solution before infusion of levcromakalim, were of the same kind as in other protocols, both in air and in hypoxia, and no correlation was observed between the baseline value of PPVR during hypoxia and any change following levcromakalim.

The summary of the effects of the various stimuli is shown in table 2. No significant decrease in the peripheral pulmonary vascular tone was observed with tested vasodilators when the pulmonary vascular tone had not been increased by prior exposure to L-NAME or during hypoxia. After an increase in PPVR, vasodilator agents (NO donor and calcium channel blockers) induced a decrease in PPVR, although PPVR remained elevated compared to baseline.

\section{Discussion}

The present results were observed in intact, though anaesthetized, animals, in a small portion of the lung vasculature, peripheral to a pulmonary artery with a $2.3 \mathrm{~mm}$ inner diameter. The amount of drug infused 
Table 2. - Peripheral pulmonary vascular resistance (PPVR) difference between control and drugs

\begin{tabular}{lclll}
\hline & \multicolumn{2}{c}{ Normoxia } & \multicolumn{2}{c}{ Hypoxia } \\
\hline L-NAME & $70 \pm 18^{* * *}(+95 \%)$ & $55 \pm 21^{* *}$ & $(+60 \%)$ \\
SNP & $-35 \pm 19^{*}$ & $(-24 \%)$ & $-30 \pm 8^{*}$ & $(-23 \%)$ \\
Verapamil & $-17 \pm 16$ & $(-12 \%)$ & $-48 \pm 37$ & $(-28 \%)$ \\
Nifedipine & $2 \pm 9$ & $(+5 \%)$ & $-31 \pm 10^{*}$ & $(-27 \%)$ \\
Nicardipine & $10 \pm 21$ & $(+12 \%)$ & $-60 \pm 40^{*}$ & $(-33 \%)$ \\
Levcromakalim & $1 \pm 14$ & $(-1 \%)$ & $+7 \pm 14$ & $(+5 \%)$ \\
\hline
\end{tabular}

Mean absolute PPVR difference $\left(10^{3} \cdot \mathrm{dyn} \cdot \mathrm{s} \cdot \mathrm{cm}^{-5}\right)$ between the highest drug concentration, and control (or L-NAME for $\mathrm{SNP}$ ). The $\%$ difference is in relation to the control (or LNAME) PPVR. SNP: sodium nitroprusside. *: $\mathrm{p}<0.05$; **: $\mathrm{p}<0.01 ; * * *: \mathrm{p}<0.001$

was too low to generate any effect on the systemic or overall pulmonary circulation. PPVR baseline values varied widely from one location to another because of local variations such as differences in the volume of the wedged vascular bed [14] but did not vary with a local infusion of glucose repeated four times in the same area, during normoxia or during hypoxia (protocol 1), showing that this procedure is suitable to study pharmacological interventions in the peripheral pulmonary circulation.

Due to the low $\mathrm{pH}$ of the control and drug solutions, minor acidosis of the blood infused during the pressure/ flow runs cannot be excluded, although a significant decrease in blood $\mathrm{pH}$ is unlikely because of the small rate of infusion (15\% of the blood infusion). Acidosis stimulates NO production, both in vitro and in vivo, leading to changes in vasomotor tone $[17,18]$. However, in vitro $\mathrm{pH}$ measurements in blood sampled from the pulmonary artery and diluted with $15 \%$ of a control solution showed a small $\mathrm{pH}$ decrease of $0.03 \pm$ $0.01(\mathrm{n}=4)$. During protocol 1 , no change in the overall pulmonary or systemic circulation, or in PPVR was observed. These results exclude significant acidosis and NO production along the experiments.

The pharmacological studies in the peripheral circulation in vivo were performed during normoxia, and during moderate hypoxia with a mean $\mathrm{Pa}_{2} \mathrm{O}_{2}$ of $45 \mathrm{mmHg}$, i.e. in the order of magnitude one might see in clinical situations such as pneumonia, acute respiratory distress syndrome or high altitude. As previously described [14], increases in PAP and PPVR during hypoxia have been observed.

The present data show that: 1) L-NAME caused an increase in the peripheral pulmonary vascular tone, potentiated the pulmonary vasoconstrictor response to hypoxia and did not suppress the vasodilation to SNP; 2) the calcium channel blocker dihydropyridines (nifedipine and nicardipine) decreased the PPVR during hypoxia; and 3) a potassium channel opener, levcromakalim, did not modify PPVR, during normoxia or acute mild hypoxia.

\section{L-NAME}

Variable effects of NO inhibitors on the normoxic pulmonary circulation have been observed. In adult animals, acute administration of arginine analogues did not influence pulmonary vascular tone in perfused dog lungs [9], in conscious dogs [8], or in intact anaesthetized dogs [19]. In other studies, however, inhibitors of NO synthesis increased normoxic pulmonary vascular tone [7]. In response to acute hypoxia, NO synthesis inhibitors have been shown to potentiate pulmonary vasoconstriction in intact anaesthetized dogs $[7,19]$. In the present study, L-NAME significantly increased normoxic and hypoxic pulmonary vascular tone. These results suggest that the background production of NO is important in the modulation of the peripheral pulmonary vascular tone in the dog.

SNP inhibited the hypoxic pulmonary vasoconstriction in anaesthetized dogs [20]. Moreover, the NO donor was an effective vasodilator of all pulmonary vessels with the exception of the smallest arteries in a model of sheep isolated pulmonary arteries and veins [21]. SNP also showed a dilator action on the pulmonary vascular pressure/flow relationship in conscious dogs after pulmonary vasoconstriction, and was not consistently affected by blockade of endogenous release by an inhibitor of NO synthesis [8]. The present results in the peripheral lung vasculature were consistent with these since, after L-NAME, SNP decreased the PPVR during normoxia and hypoxia, and confirmed that exogenous NO was able to induce a decrease in the peripheral pulmonary vascular tone [21].

\section{Calcium blockers}

The effects of verapamil, nifedipine and nicardipine on the PPVR were dependent on vascular tone: these drugs were ineffective in normoxia, and during acute hypoxia the vasodilation they produced was related to the increased resistance value. However, vasodilator action did reach statistical significance for dihydropyridines only. With verapamil during hypoxia, PPVR seemed to decrease, but the changes were not significant because of the dispersion of the results. In the literature, verapamil had no significant effect on the pulmonary vascular tone during normoxia in isolated pulmonary artery rings [22] nor in anaesthetized dogs. Similarly nifedipine had no significant effect on tone during normoxia in anaesthetized dogs [13]. During acute $\mathrm{HPV}$, various studies have reported effective vasodilating actions of calcium channel inhibitors. During hypoxia, verapamil inhibited HPV in isolated rat lungs [12] but not in human pulmonary artery rings [22]. Although verapamil did not induce any significant change in the pulmonary vascular tone in anaesthetized dogs, nifedipine decreased pulmonary vascular resistance [13]. In the peripheral pulmonary vasculature, the present study observed that dihydropyridines decreased the enhanced hypoxic pulmonary vascular tone.

\section{Levcromakalim}

The present findings did not confirm a vasodilating action of the potassium $\left(\mathrm{K}^{+}\right)$channel opener levcromakalim in the peripheral pulmonary circulation. Levcromakalim, the active enantiomer of cromaka$\mathrm{lim}$, is a $\mathrm{K}^{+}$channel opener that activates adenosine 
triphosphate (ATP)-sensitive $\mathrm{K}^{+}$channels $\left(\mathrm{K}_{\mathrm{ATP}}\right.$ channels) in vascular smooth muscle [23]. Several studies suggest the role of $\mathrm{K}^{+}$channel inhibition in HPV [10, 24]. Levcromakalim has a relaxant activity in $\mathrm{K}^{+}$precontracted pulmonary vessels [25], and cromakalim causes vasorelaxation on HPV [24]. The low basal pulmonary vascular tone could explain the ineffectiveness of levcromakalim in our study in normoxia but not in hypoxia, because hypoxia increases PPVR [14]. Moreover, this study's baseline PPVR values, during hypoxia, before levcromakalim infusion, were in the same order of magnitude as those observed in other protocols where calcium channel blockers and SNP were effective.

The fact that contrasting data have been observed regarding the effects of various drugs on the pulmonary vascular tone may be due to both the diversity in experimental conditions and the regional vasoreactivity. KEMP et al. [21] recently demonstrated in sheep that the reactivity to many vasoconstrictors and vasodilators was not uniform along isolated pulmonary vessels. In conduit arteries, the calcium activated $\mathrm{K}^{+}$channels ( $\mathrm{K}_{\mathrm{Ca}}$ channels) are predominant. No $\mathrm{K}_{\mathrm{ATP}}$ cell was identified in resistance arteries which have a majority of voltage-gated potassium $\left(\mathrm{K}_{\mathrm{v}}\right)$ channels [26]. Nine families of $K_{v}$ channels are recognized from cloning studies $\left(\mathrm{K}_{\mathrm{v} 1}-\mathrm{K}_{\mathrm{v} 9}\right)$, each with subtypes. The contribution of an individual $\mathrm{K}_{\mathrm{v}}$ channel to the whole cell current is difficult to determine pharmacologically because $K_{v}$ channel inhibitors are nonspecific. Using anti- $K_{\mathrm{V}}$ antibodies to immunolocalize and inhibit $\mathrm{K}_{\mathrm{V}}$ channels, ARCHER et al. [27] showed that the pulmonary arterial smooth muscle cell contains numerous types of $\mathrm{K}_{\mathrm{v}}$ channels among which $\mathrm{K}_{\mathrm{v}} 2.1$ and $\mathrm{K}_{\mathrm{v}} 1.5$ contribute to the initiation of HPV. The present experiments operated on a peripheral portion of the pulmonary vascular bed where the arteries are mainly resistance arteries. As we did not find any activity of $\mathrm{K}_{\mathrm{ATP}}$ channel openers, these results are consistent with those of ARCHER et al. [26].

The comparison of PPVR differences with the vasodilators and vasoconstrictor agents studied here in vivo, demonstrated that, in the present model, the peripheral pulmonary vasoreactivity to vasodilator agents was moderate. The low basal pulmonary vascular tone could explain the absence of effects from vasodilator drugs when pulmonary vascular tone had not been previously increased, and their moderate effects despite increased pulmonary vascular tone by mild acute hypoxia and vasoconstrictive drugs. Even during acute hypoxia, the peripheral pulmonary vasculature failed to totally relax in response to calcium channel blockers, as in previous works $[21,28]$. The reason for this small response is unclear, but it was also observed with $\mathrm{NO}$ and $\beta$-adrenoceptor-mediated relaxation in other models [21].

In conclusion, the effects of several vasoactive drugs on the peripheral pulmonary circulation without any effect on the systemic or overall pulmonary circulation, in vivo, in anaesthetized intact dogs, during normoxia and during mild acute normocapnic hypoxia have been reported. This study has also demonstrated that the unstressed peripheral pulmonary vasculature displayed little reactivity to vasodilator drugs. These results underline the role of the nitric oxide pathway, and suggest the lack of functional adenosine triphosphate sensitive potassium channels in peripheral pulmonary circulation during normoxia and during acute hypoxia. The role of calcium channels seems essential during mild acute hypoxia in the peripheral lung vasculature. One of the possible implications in clinical practice would be the pharmacological decrease in peripheral pulmonary vascular resistance during hypoxia, in agreement with recent studies showing beneficial effects of calcium channel blockers during high altitude pulmonary oedema [29]. This model could help to select new drugs such as other potassium channel openers or endothelin receptor antagonists that could be useful in clinical practice [30].

Acknowledgements. The authors thank J. Atkinson for critical review of the manuscript, F. Poincelot and J. Lambert for skillful assistance, B. Clement and P. Ulmer for typing the manuscript, and M.C. Rohrer for drawing the figures.

\section{References}

1. Madden JA, Vadula MS, Kurup VP. Effects of hypoxia and other vasoactive agents on pulmonary and cerebral artery smooth muscle cells. Am J Physiol 1992; 263: L384-L393.

2. Bennie RE, Packer CS, Powell DR, Jin N, Rhoades RA. Biphasic contractile response of pulmonary artery to hypoxia. Am J Physiol 1991; 261: L156-L163.

3. Kadowitz PJ, Lippton HL, Bellan JA, Hyman AL. Nisoldipine inhibits vasoconstrictor responses in the cat pulmonary vascular bed. J Appl Physiol 1989; 66: 2885-2890.

4. Moncada S, Palmer RMJ, Higgs EA. Nitric oxide: physiology, pathophysiology, and pharmacology. Pharmacol Rev 1991; 43: 109-142.

5. Zhao YJ, Wang J, Rubin LJ, Yuan, XJ. Inhibition of $\mathrm{K}_{\mathrm{v}}$ and $\mathrm{K}_{\mathrm{Ca}}$ channels antagonizes NO-induced relaxation in pulmonary artery. Am J Physiol 1997; 272: H904-H912.

6. Archer S, Tolins J, Raij L, Weir E. Hypoxic pulmonary vasoconstriction is enhanced by inhibition of the synthesis of an endothelium derived relaxing factor. Biochem Biophys Res Commun 1989; 164: 1198-1205.

7. Perella MA, Edell ES, Krowka MJ, Corteses DA, Burnett JC Jr. Endothelium-derived relaxing factor in pulmonary and renal circulations during hypoxia. $\mathrm{Am}$ J Physiol 1992; 263: R45-R50.

8. Nishiwaki $\mathrm{K}$, Nyhan DP, Rock $\mathrm{P}$, et al. $\mathrm{N}^{\omega}$-nitro-Larginine and pulmonary vascular pressure-flow relationship in conscious dogs. Am J Physiol 1992; 262: H1331-H1337.

9. Barnard JW, Wilson PS, Moore TM, Thompson WJ, Taylor AE. Effect of nitric oxide and cyclooxygenase products on vascular resistance in dog and rat lungs. J Appl Physiol 1993; 74: 2940-2948.

10. Post JM, Hume JR, Archer SL, Weir EK. Direct role for potassium channel inhibition in hypoxic pulmonary vasoconstriction. Am J Physiol 1992; 262: C882C890.

11. Weir EK, Archer SL. The mechanism of acute hypoxic 
pulmonary vasoconstriction: the tale of two channels. FASEB J 1995; 9: 183-189.

12. McMurtry IF, Davidson AB, Reeves JT, Grover RF. Inhibition of hypoxic pulmonary vasoconstriction by calcium antagonists in isolated rat lungs. Circ Res 1976; 38: 99-104.

13. Young TE, Lundquist LJ, Chesler E, Weir EK. Comparative effects of nifedipine, verapamil, and diltiazem on experimental pulmonary hypertension. $\mathrm{Am} \mathrm{J}$ Cardiol 1983; 51: 195-200.

14. Schrijen F, Saunier C, Chabot F. Peripheral pulmonary vascular resistance. J Appl Physiol 1993; 74: 613616.

15. Chabot F, Saunier C, Schrijen F. Changes in peripheral pulmonary vascular resistance after ioxaglate infusion in anesthetized dogs. Can J Physiol Pharmacol 1997; 75: 15-18.

16. Snedecor GW, Cochran WG. Chapter 7: Correlation; Chapter 10: Analysis of variance in two or more groups of measurement data. In: Snedecor GW, Cochran WG. Statistical methods. Ames, Iowa State University Press, 1967; pp. 138-168; 214-252.

17. Zweier JL, Wang P, Samouilov A, Kuppusamy P. Enzyme-independent formation of nitric oxide in biological tissues. Nat Med 1995; 1: 804-809.

18. Pedoto A, Caruso JE, Nandi J, et al. Acidosis stimulates nitric oxide production and lung damage in rats. Am J Respir Crit Care Med 1999; 159: 397-402.

19. Leeman M, Zegers de Beyl V, Delcroix M, Naeije R. Effects of endogenous nitric oxide on pulmonary vascular tone in intact dogs. Am J Physiol 1994; 266: H2343-H2347.

20. Naeije R, Lejeune P, Leeman M, Melot C, Deloof T. Pulmonary arterial pressure-flow plots in dogs: effects of isoflurane and nitroprusside. J Appl Physiol 1987; 63: 969-977.

21. Kemp BK, Smolich JJ, Cocks TM. Evidence for specific regional patterns of responses to different vasoconstrictors and vasodilators in sheep isolated pulmonary arteries and veins. Br J Pharmacol 1997; 121: 441-450.

22. Demiryurek AT, Wadsworth RM, Kane KA, Peacock AJ. The role of endothelium in hypoxic constriction of human pulmonary artery rings. Am Rev Respir Dis 1993; 147: 283-290.

23. Nelson MT, Quayle JM. Physiological roles and properties of potassium channels in arterial smooth muscle. Am J Physiol 1995; 268: C799-C822.

24. Barman SA. Potassium channels modulate hypoxic pulmonary vasoconstriction. Am J Physiol 1998; 275: L64-L70.

25. Dumas M, Dumas JP, Advenier C, Giudicelli JF. Effect of three $\mathrm{K}^{+}$channel openers on airways and pulmonary circulation in the isolated guinea-pig lung. Eur J Pharmacol 1993; 239: 141-147.

26. Archer SL, Huang JMC, Reeve HL, et al. Differential distribution of electrophysiologically distinct myocytes in conduit and resistance arteries determines their response to nitric oxide and hypoxia. Circ Res 1996; 78: 431-442.

27. Archer SL, Souil E, Dinh-Xuan AT, et al. Molecular identification of the role of voltage-gated $\mathrm{K}^{+}$channels, $\mathrm{Kv}_{1.5}$ and $\mathrm{Kv}_{2.1}$, in hypoxic pulmonary vasoconstriction and control of resting membrane potential in rat pulmonary artery myocytes. J Clin Invest 1998; 101: 2319-2330.

28. Leach RM, Twort CHC, Cameron IR. The mechanism of action of endothelin-1 on small pulmonary arterial vessels. Pulm Pharmacol 1990; 3: 103-109.

29. Watt M, Peacock AJ, Newell J, McDonagh T, Grant $\mathrm{S}$. The effect of amlodipine on respiratory and pulmonary vascular responses to hypoxia in mountaineers. Eur Respir J 2000; 15: 459-463.

30. Dumas JP, Bardou M, Goirand F, Dumas M. Hypoxic pulmonary vasoconstriction. Gen Pharmacol 1999; 33 : 289-297. 